\title{
INDUCED UNIVERSAL MAPS AND SOME HYPERSPACES WITH THE FIXED POINT PROPERTY
}

SAM B. NADLER, JR.

\begin{abstract}
For a (metric) continuum $Z$, let $2^{Z}$ (resp., $C(Z)$ ) denote the space of all nonempty compacta (resp., continua) in $Z$ with the Hausdorff metric. We prove: (1) If $f$ is a monotone map of a continuum $X$ onto a Peano continuum $Y$, then, for any maps $g: 2^{X} \rightarrow 2^{Y}$ and $h: C(X) \rightarrow C(Y)$, there is $A \in 2^{X}$ and $B \in C(X)$ such that $f(A)=g(A)$ and $f(B)=h(B)$. We use (1) to prove: (2) If $X$ is an inverse limit of dendrites with quasi-monotone bonding maps, then $2^{X}$ and $C(X)$ have the fixed point property. Thus, we have a proof that for certain indecomposable continua $X, 2^{X}$ has the fixed point property.
\end{abstract}

1. Introduction. A continuum is a nonempty compact connected metric space. The hyperspaces of a continuum $X$ are $2^{X}=\{A \subset X: A$ is nonempty and compact $\}$ and $C(X)=\left\{A \in 2^{X}: A\right.$ is connected $\}$ with the Hausdorff metric [11]. A map is a continuous function. A map $f: X \rightarrow Y$ is universal [6] provided that for any map $g: X \rightarrow Y$ there is a point $p \in X$ such that $f(p)=g(p)$.

It is of interest to know conditions under which a map is universal and to have nontrivial examples of universal maps (e.g., see [4-7 and 12]). Applications of such results are in fixed point theory and the structure of continua. For example: In 2.11 of $[12]$ we showed that a certain induced map is universal; then we obtained Segal's fixed point theorem (Theorem 3 of [15]) and Read's theorem about maps onto arc-like continua (Theorem 4 of [14]) as immediate corollaries. We remark that universal maps are used in a disguised form in dimension theory-the AlexanderHopf maps onto $n$-cells are precisely the universal maps onto $n$-cells (1.3 of [12]). Thus, universal maps may be viewed as a generalization to arbitrary range spaces of Alexander-Hopf maps.

If $X$ and $Y$ are continua and $f: X \rightarrow Y$ is a map, then there are two induced maps, $f^{*}: 2^{X} \rightarrow 2^{Y}$ and $\hat{f}: C(X) \rightarrow C(Y)$, defined by $f^{*}(A)=\{f(a): a \in A\}$ for all $A \in 2^{X}$ and $\hat{f}=f^{*} \mid C(X)$.

In this paper we show that $f^{*}$ and $\hat{f}$ are universal when $f$ is any monotone map from a continuum onto a Peano continuum (2.3), and we use this result to show that $2^{X}$ and $C(X)$ have the fixed point property for certain continua $X$ (see $\S 3)$. One of the consequences of our work is that for the first time the fixed point property has been verified for $2^{X}$ when $X$ is some indecomposable continuum (3.5). We also mention that the result in (2.3) and [3] can be used to construct interesting

Received by the editors October 7, 1985 and, in revised form, January 27, 1986.

1980 Mathematics Subject Classification (1985 Revision). Primary 54B20, 54C10; Secondary $54 \mathrm{~F} 20$.

Key words and phrases. $C E$-map, continuum, dendrite, fixed point property, hyperspaces, indecomposable continuum, inverse limit, monotone map, Peano continuum, quasi-monotone map, universal map. 
examples of universal maps onto Hilbert cubes. Such examples have been lacking in the past.

We assume that the reader is familiar with the concepts which are intrinsic to continua theory and hyperspaces. We will provide references at appropriate places in the paper. Nonetheless, we recall the following few definitions. A map is monotone provided that each point-inverse is connected [16, p. 70]. A CE-map is a map such that each point-inverse has trivial shape in the sense of Borsuk [2]. A Peano continuum is a locally connected continuum. If $f: X \rightarrow Y$ is a map and $A \subset X$, then $f \mid A$ denotes the restriction of $f$ to $A$.

2. Induced universal maps. The main result in this section, (2.3), is proved using the following two lemmas, the first of which is related to Theorem 1.1 of [10].

(2.1) LemMA. For any two continua $X$ and $Y$ and a mapping $f: X \rightarrow Y$, the following three statements are equivalent:

(1) $f$ is a monotone map from $X$ onto $Y$;

(2) $f^{*}$ is a CE-map from $2^{X}$ onto $2^{Y}$,

(3) $\hat{f}$ is a $C E$-map from $C(X)$ onto $C(Y)$.

ProOF. We first prove that (1) implies (2). Assume that (1) holds. Let $B \in 2^{Y}$. Let $\Lambda=\left(f^{*}\right)^{-1}(B)$. We must show that $\Lambda$ has trivial shape. We first show that $\Lambda$ is a continuum. Let $L=f^{-1}(B)$. Since $f$ maps $X$ onto $Y, f(L)=B$; thus, $f^{*}(L)=B$. Hence, $L \in \Lambda$. Thus, $\lambda \neq \varnothing$. Since $f^{*}$ is continuous, $\Lambda$ is compact. Now, to see that $\Lambda$ is connected, let $A \in \Lambda$ and assume that $A \neq L$. We show that there is an arc in $\Lambda$ from $A$ to $L$. Since $A \in \Lambda, f(A)=B$. Hence, by the definition of $L$,

(a) $A \subset L$.

Let $L_{1}$ be a component of $L$. Then $f\left(L_{1}\right)$ is contained in a component $B_{1}$ of $B$. Note that $L_{1} \subset f^{-1}\left(B_{1}\right) \subset L$ and that, since $f$ is monotone, $f^{-1}\left(B_{1}\right)$ is connected. Thus, since $L_{1}$ is a component of $L, L_{1}=f^{-1}\left(B_{1}\right)$. Therefore, since $f(A)=B$, $A \cap L_{1} \neq \varnothing$. Hence, we have proved that

(b) each component of $L$ intersects $A$.

By (a), (b), and 1.8 of $\left[\mathbf{1 1}\right.$, p. 59] there is an order arc $\Gamma$ in $2^{X}$ from $A$ to $L$. Since $f(A)=B=f(L)$ and since, for all $G \in \Gamma, A \subset G \subset L$, it follows easily that $\Gamma \subset \Lambda$. Thus, it follows that we have proved $\Lambda$ is (arcwise) connected. Therefore, $\Lambda$ is a continuum. Hence, by 1.182 of $[11$, p. 180], $C(\Lambda)$ has trivial shape. For each $\alpha \in C(\Lambda)$, let $\sigma(\alpha)=\bigcup \alpha$. By 1.48 of $[11$, p. 100], $\sigma$ is continuous on $C(\Lambda)$. It is easy to see the $\sigma$ maps $C(\Lambda)$ to $\Lambda$ and that for all $E \in \Lambda, \sigma(\{E\})=E$. Hence, $\sigma$ is an $r$-map [1, p. 18] from $C(\Lambda)$ onto $\Lambda$. Therefore, since having trivial shape is an $a$ invariant under $r$-maps [2, p. 103], $\Lambda$ has trivial shape. Thus, we have proved that (1) implies (2).

The proof that (1) implies (3) is similar to the proof that (1) implies (2). However, there are some modifications which are worth noting. Let $B \in C(Y)$, $\Lambda=\hat{f}^{-1}(B), L=f^{-1}(B)$, and $A \in \Lambda$ such that $A \neq L$. Since $\Lambda \subset C(X)$, the monotoneness of $f$ must be invoked to see that $L \in \Lambda$. As above, (a) holds. Since $L$ is connected, (b) follows immediately from (a). Hence, again the order arc $\Gamma$ in $2^{X}$ from $A$ to $L$ exists; however, to see that $\Gamma \subset \Lambda$, we must use 1.11 of $[11$, p. 64] to know that $\Gamma \subset C(X)$. It then follows as above that $\Lambda$ is a continuum. Define $\sigma$ on $C(\Lambda)$ as above. To see that $\sigma$ maps $C(\Lambda)$ to $\Lambda$ we must use 1.43 of $[\mathbf{1 1}$, p. 97] in 
order to know that $\sigma(\alpha) \in C(X)$ for all $\alpha \in C(\Lambda)$. It then follows as above that $\sigma$ is an $r$-map from $C(\Lambda)$ onto $\Lambda$ and, thus, that $\hat{f}$ is a $C E$-map.

Next we prove that (2) implies (1). Assume that (2) holds. Let $y \in Y$. Since $f^{*}$ is a $C E$-map, $f^{*}$ is monotone. Hence, $\left(f^{*}\right)^{-1}(\{y\})$ is connected. Thus, since

$$
\left(f^{*}\right)^{-1}(\{y\})=2^{f^{-1}(y)},
$$

$2^{f^{-1}(y)}$ is connected. Hence, $f^{-1}(y)$ is connected. Therefore, $f$ is monotone. Since $f^{*}$ maps $2^{X}$ onto $2^{Y}, f$ must map $X$ onto $Y$. This completes the proof that $(2)$ implies (1).

Finally, the proof that (3) implies (1) is similar to the proof that (2) implies (1) using $\hat{f}^{-1}(\{y\})=C\left(f^{-1}(y)\right)$.

(2.2) LEMMA. Let $f$ be a monotone map from a continuum $X$ onto a continuum $Y$. Then there are Peano continua $X_{i}$ and $Y_{i}, i=1,2, \ldots$, and a map $F: X_{1} \rightarrow Y_{1}$ such that $X_{i} \supset X_{i+1}$ and $Y_{i} \supset Y_{i+1}$ for each $i, \bigcap_{i=1}^{\infty} X_{i}=X, \bigcap_{i=1}^{\infty} Y_{i}$ $=Y, F \mid X_{i}$ is a monotone map of $X_{i}$ onto $Y_{i}$ for each $i$, and $F \mid X=f$.

Proof. For each $n=1,2, \ldots$, let $I_{n}=[0,1]$. Let $Q=\prod_{n=1}^{\infty} I_{n}$ with the cartesian product metric $d$ given by

$$
d\left(\left(u_{n}\right)_{n=1}^{\infty},\left(v_{n}\right)_{n=1}^{\infty}\right)=\sum_{n=1}^{\infty} 2^{-n}\left|u_{n}-v_{n}\right|
$$

for all $\left(u_{n}\right)_{n=1}^{\infty},\left(v_{n}\right)_{n=1}^{\infty} \in Q$. Consider $X$ and $Y$ as lying in $Q$ such that for all $\left(z_{n}\right)_{n=1}^{\infty} \in X \cup Y, z_{n}=0$ for all $n=2,4,6, \ldots\left[9\right.$, p. 23]. For any $u=\left(u_{n}\right)_{n=1}^{\infty}$ and $v=\left(v_{n}\right)_{n=1}^{\infty}$ in $X \cup Y$, and for any $j=1,2, \ldots$, we define $\langle u, v ; j\rangle$ as follows: If $u \neq v$, then $\langle u, v ; j\rangle$ is the arc in $Q$ from $u$ to $v$ defined by

$$
\begin{aligned}
& \langle u, v ; j\rangle=\left\{\left(q_{n}\right)_{n=1}^{\infty} \in Q: \text { for some } t \in[0,1],\right. \\
& \left.\qquad q_{n}=(1-t) u_{n}+t v_{n} \text { for all } n \neq 2 j \text { and } q_{2 j}=t-t^{2}\right\}
\end{aligned}
$$

and, if $u=v$, then

$$
\langle u, v ; j\rangle=\{u\}
$$

Let $C$ denote the Cantor middle-thirds set in $[0,1]$, and let $\left(a_{j}, b_{j}\right), j=1,2, \ldots$, be a one-to-one enumeration of the components of $[0,1]-C$. Let $g$ be a map of $C$ onto $X[9$, p. 23]. Then, for each $i=1,2, \ldots$, let

$$
X_{i}=X \cup\left(\bigcup_{j=i}^{\infty}\left\langle g\left(a_{j}\right), g\left(b_{j}\right) ; j\right\rangle\right)
$$

and

$$
Y_{i}=Y \cup\left(\bigcup_{j=i}^{\infty}\left\langle f\left(g\left(a_{j}\right)\right), f\left(g\left(b_{j}\right)\right) ; j\right\rangle\right) .
$$

Next we define $F: X_{1} \rightarrow Y_{1}$. For each $j=1,2, \ldots$, define a map $F_{j}$ on $\left\langle g\left(a_{j}\right), g\left(b_{j}\right)\right.$; $j\rangle$ as follows. If $f\left(g\left(a_{j}\right)\right) \neq f\left(g\left(b_{j}\right)\right)$, then let $F_{j}$ be a homeomorphism of the arc $\left\langle g\left(a_{j}\right), g\left(b_{j}\right) ; j\right\rangle$ onto the arc $\left\langle f\left(g\left(a_{j}\right)\right), f\left(g\left(b_{j}\right)\right) ; j\right\rangle$ such that $F_{j}$ agrees with $f$ at the 
two points $g\left(a_{j}\right)$ and $g\left(b_{j}\right)$. If $f\left(g\left(a_{j}\right)\right)=f\left(g\left(b_{j}\right)\right)$, then let $F_{j}$ map $\left\langle g\left(a_{j}\right), g\left(b_{j}\right) ; j\right\rangle$ onto the one-point set $\left\langle f\left(g\left(a_{j}\right)\right), f\left(g\left(b_{j}\right)\right) ; j\right\rangle$. Now define $F: X_{1} \rightarrow Y_{1}$ by

$$
F(p)= \begin{cases}f(p), & \text { if } p \in X, \\ F_{j}(p), & \text { if } p \in\left\langle g\left(a_{j}\right), g\left(b_{j}\right) ; j\right\rangle .\end{cases}
$$

We note that he $\operatorname{arcs}$ joined to $X$ to obtain $X_{1}$ only intersect $X$ in their endpoints, are mutualy disjoint except possibly for endpoints, and form a null sequence with respect to the metric $d$. The same is true of the arcs joined to $Y$ to obtain $Y_{1}$. Using these observations it follows that $F$ is a function and is continuous. It also follows that $g$ can be extended to a map $G$ of $[0,1]$ onto $X_{1}$ such that for each $i>1$, $G$ maps $[0,1]-\bigcup_{j=1}^{i-1}\left(a_{j}, b_{j}\right)$ onto $X_{i}$. Hence, each $X_{i}$ is a Peano continuum. Thus, since $F\left(X_{i}\right)=Y_{i}$, each $Y_{i}$ is a Peano continuum. The monotonicity of $F \mid X_{i}$ for each $i$ follows using the monotonicity of $f$ and the observations made above about the arcs added to $Y$ (also note that any two different $\operatorname{arcs}$ in $X_{i}$ go to different arcs in $Y_{i}$ under $\left.F \mid X_{i}\right)$. The other properties claimed in the lemma are clearly sataisfied by the construction.

Now we prove the following main theorem.

(2.3) THEOREM. If $f$ is a monotone map from a continuum $X$ onto a Peano continuum $Y$, then $f^{*}: 2^{X} \rightarrow 2^{Y}$ and $\hat{f}: C(X) \rightarrow C(Y)$ are universal.

PROOF. We prove the theorem for $\hat{f}$, the proof for $f^{*}$ being similar. Let $g$ be any map from $C(X)$ into $C(Y)$. Let $X_{i}, Y_{i}$, and $F$ be as in (2.2). By 1.96 of [11, p. 136], $g$ can be extended to a mapping $G: C\left(X_{1}\right) \rightarrow C(Y)$. Fix $i$. By $(2.2), X_{i}$ and $Y_{i}$ are Peano continua and $F \mid X_{i}=F_{i}$ is a monotone map of $X_{i}$ onto $Y_{i}$. Hence, by 1.96 of $\left[11\right.$, p. 136], $C\left(X_{i}\right)$ and $C\left(Y_{i}\right)$ are absolute retracts and, by $(2.1), \hat{F}_{i}$ is a $C E$-map from $C\left(X_{i}\right)$ onto $C\left(Y_{i}\right)$. Thus, by 3.10 of $\left[\mathbf{1 2}\right.$, p. 233], $\hat{F}_{i}$ is universal. Therefore, since $G \mid C\left(X_{i}\right)$ maps $C\left(X_{i}\right)$ into $C\left(Y_{i}\right)$, there exists $A_{i} \in C\left(X_{i}\right)$ such that $\hat{F}_{i}\left(A_{i}\right)=G\left(A_{i}\right)$. Note that since $X_{i} \subset X_{1}, A_{i} \in C\left(X_{1}\right)$ and $\hat{F}\left(A_{i}\right)=G\left(A_{i}\right)$. We have shown that such an $A_{i}$ exists for each $i=1,2, \ldots$ Now, since $C\left(X_{1}\right)$ is compact $[11$, p. 7$]$, the sequence $\left\{A_{i}\right\}_{i=1}^{\infty}$ has a convergent subsequence $\left\{A_{i(k)}\right\}_{k=1}^{\infty}$. Let $A=\lim _{k \rightarrow \infty} A_{i(k)}$. Then, since $\hat{F}\left(A_{i(k)}\right)=G\left(A_{i(k)}\right)$ for each $k$, it follows from the continuity of $\hat{F}$ and $G$ that $\hat{F}(A)=G(A)$. Since $A_{i(k)} \in C\left(X_{i(k)}\right)$ for each $k$ and since $\bigcap_{k=1}^{\infty} X_{i(k)}=X$ (by $(2.2)$ ), we see that $A \in C(X)$. Hence, $G(A)=g(A)$ and, since $F \mid X=f$ (by $(2.2)$ ), $\hat{F}(A)=\hat{f}(A)$. Therefore, $\hat{f}(A)=g(A)$ and we have proved $\hat{f}$ is universal.

(2.4) COROLlaRY. If $f$ is a monotone map from a continuum $X$ onto a Peano continuum $Y$ and $g$ is any map from $X$ into $Y$, there is a subcontinuum $A$ of $X$ such that $f(A)=g(A)$.

REMARKS. 1. The condition in (2.3) that $Y$ be a Peano continuum is necessary. In particular, since all continua with trivial shape are nested intersections of ARs (2.1 of [8]), it may appear from the nature of the constructions in the proofs of (2.2) and (2.3) that (2.3) is true for all continua $Y$ with trivial shape. However, this is false even when $f$ is a universal homeomorphism. To see this, let $f$ be the identity map on the continuum $Y$ in $[\mathbf{1 3}]$ and apply the Theorem in $[\mathbf{1 3}$, p. 256] to $\hat{f}=$ idy on $C(Y)$. 
2. The condition in (2.3) that $f$ be monotone cannot be weakened to require only that $f$ be nonalternating even when $X$ and $Y$ are one-dimensional Peano continua. For example, let $f$ be the natural map of the unit circle $S^{1}$ onto a figure eight $E$ which identifies the points $(0,1)$ and $(0,-1)$. Then $f$ is nonalternating [16, p. 127] but, since $\hat{f}$ does not map $C\left(S^{1}\right)$ onto $C(E), \hat{f}$ is not universal. It would be of interest to know if (2.3) remains valid for quasi-monotone maps [16, p. 151]. By using Proposition 5 of [6] and (3.2) below it can be seen easily that (2.3) remains true for quasi-monotone maps of Peano continua onto dendrites.

3. Some hyperspaces with the fixed point property. It is of interest to know when $2^{X}$ and $C(X)$ have the fixed point property (see Chapter VII of $[\mathbf{1 1}]$ ). We use (2.3) to prove the general result in (3.1), and then we give some specific consequences of this result.

The notation $X=\lim \left\{X_{i}, f_{i}\right\}$ means $X$ is an inverse limit of continua $X_{i}$ with bonding maps $f_{i}: X_{i+1} \rightarrow X_{i}$ for each $i=1,2, \ldots$ For any $j$ and $k$ with $j \leq k$, we let $f_{j k}$ denote the composition $f_{j} \circ \cdots \circ f_{k-1} \circ f_{k}$ if $j<k$ and, if $j=k, f_{j j}=f_{j}$.

(3.1) Proposition. Let $X=\lim \left\{X_{i}, f_{i}\right\}$ where $X_{i}$ is a Peano continuum for each $i=1,2, \ldots$ Assume that for each $i$ there is a subcontinuum $Y_{i+1}$ of $X_{i+1}$ such that $f_{i} \mid Y_{i+1}$ maps $Y_{i+1}$ monotonely onto $X_{i}$. Then, $2^{X}$ and $C(X)$ have the fixed point property.

ProOF. We prove the theorem for $2^{X}$, the proof for $C(X)$ being similar. It follows easily that for each $j \leq k$ there is a subcontinuum $Z_{k+1}$ of $X_{k+1}$ such that $f_{j k} \mid Z_{k+1}$ maps $Z_{k+1}$ monotonely onto $X_{j}$. By $(2.3)$, each $\left(f_{j k} \mid Z_{k+1}\right)^{*}: 2^{Z_{k+1}} \rightarrow$ $2^{X_{j}}$ is universal. Thus, since each $\left(f_{j k} \mid Z_{k+1}\right)^{*}=f_{j k}^{*} \mid 2^{Z_{k+1}}$, each $f_{j k}^{*} \mid 2^{Z_{k+1}}: 2^{Z_{k+1}}$ $\rightarrow 2^{X_{j}}$ is universal. Hence, clearly (Proposition 5 of [6]), each $f_{j k}^{*}: 2^{X_{k+1}} \rightarrow 2^{X_{j}}$ is universal. Thus, since each $2^{X_{i}}$ is an $\operatorname{AR}\left(1.96\right.$ of $\left[\mathbf{1 1}\right.$, p. 136]), $\lim \left\{2^{X_{i}}, f_{i}^{*}\right\}$ has the fixed point property (Corollary 1 of $[6$, p. 437]). Therefore, by 1.169 of [11, p. 171], $2^{X}$ has the fixed point property.

Recall that a map $f$ from $X$ onto $Y$ is quasi-monotone provided that for any continuum $K$ in $Y$ with nonempty interior, $f^{-1}(K)$ has only a finite number of components and each of these maps onto $K$ under $f[16$, p. 151]. A dendrite is a Peano continuum containing no simple closed curve $[\mathbf{1 6}$, p. 88].

(3.2) Lemma. If $f: X \rightarrow D$ is a quasi-monotone map from a Peano continuum $X$ onto a dendrite $D$, then there is a subcontinuum $Z$ of $X$ such that $f \mid Z: Z \rightarrow D$ maps $Z$ monotonely onto $D$.

PrOOF. By 8.4 of [16, p. 153], there is a monotone map $m$ from $X$ onto a continuum $M$ and a light open map $l$ from $M$ onto $D$ such that $f=l \circ \mathrm{m}$. By 2.4 of $[16, \mathrm{p} .188]$ there is a dendrite $E$ in $M$ such that $l \mid E$ is a homeomorphism of $E$ onto $D$. Taking $Z=m^{-1}(E)$, it follows easily that $f \mid Z$ maps $Z$ monotonely onto D.

(3.3) THEOREM. If $X=\lim \left\{D_{i}, f_{i}\right\}$, where $D_{i}$ is a dendrite and $f_{i}$ is a quasimonotone map of $D_{i+1}$ onto $D_{i}$ for each $i=1,2, \ldots$, then $2^{X}$ and $C(X)$ have the fixed point property.

ProOF. Use (3.2) to apply (3.1). 
(3.4) COROllaRY. If $X=\lim \left\{D_{i}, f_{i}\right\}$, where $D_{i}$ is a dendrite and $f_{i}$ is an open map of $D_{i+1}$ onto $D_{i}$ for each $i=1,2, \ldots$, then $2^{X}$ and $C(X)$ have the fixed point property.

PROOF. Since open maps between Peano continua are quasi-monotone (8.11 of $[16$, p. 152]), (3.4) follows immediately from (3.3).

(3.5) REMARK. The results above can be used to show that $2^{X}$ has the fixed point property for certain indecomposable continua $X$. For example, let $X=\lim _{\longleftarrow}\left\{X_{i}, f_{i}\right\}$, where each $X_{i}=[0,1]$ and each $f_{i}$ is given by

$$
f_{i}(t)= \begin{cases}2 t, & 0 \leq t \leq 1 / 2 \\ -2 t+2, & 1 / 2 \leq t \leq 1\end{cases}
$$

Then $X$ is Knaster's indecomposable continuum [9, p. 204] and, by (3.4), $2^{X}$ has the fixed point property. Let us note that $X$ is an arc-like continuum. It is still not known if $2^{X}$ has the fixed point property for all arc-like continua (see 7.10 of [11, p. 299]).

\section{REFERENCES}

1. K. Borsuk, Theory of retracts, Monografie Math., vol. 44, Polish Sci. Publ., Warszawa, Poland, 1967.

2. Theory of shape, Monografie Mat., vol. 59, Polish Sci. Publ., Warszawa, Poland, 1975.

3. D. W. Curtis and R. M. Schori, Hyperspaces of Peano continua are Hilbert cubes, Fund. Math. 101 (1978), 19-38.

4. W. Holsztyński, On the composition and products of universal mappings, Fund. Math. 64 (1969), 181-188.

5. $\ldots$, On the product and composition of universal mappings of manifolds into cubes, Proc. Amer. Math. Soc. 58 (1976), 311-314.

6. __ Universal mappings and fixed point theorems, Bull. Acad. Polon. Sci. 15 (1967), 433-438.

7. _ Universality of mappings onto the products of snake-like spaces, Bull. Acad. Polon. Sci. 16 (1968), 161-167.

8. J. Krasinkiewicz, Curves which are continuous images of tree-like continua are movable, Fund. Math. 89 (1975), 233-260.

9. K. Kuratowski, Topology, vol. II, Academic Press, New York and London, 1968.

10. A. Y. W. Lau, A note on monotone maps and hyperspaces, Bull. Acad. Polon. Sci. 24 (1976), 121-123.

11. S. B. Nadler, Jr., Hyperspaces of sets, Dekker, New York and Basel, 1978.

12. Universal mappings and weakly confluent mappings, Fund. Math. 110 (1980), 221-235.

13. S. B. Nadler, Jr., and J. T. Rogers, Jr., A note on hyperspaces and the fixed point property, Colloq. Math. 25 (1972), 255-257.

14. D. R. Read, Confluent and related mappings, Colloq. Math. 29 (1974), 233-239.

15. J. Segal, A fixed point theorem for the hyperspace of a snake-like continuum, Fund. Math. 50 (1962), 237-248.

16. G. T. Whyburn, Analytic topology, Amer. Math. Soc. Colloq. Publ., vol. 28, Amer. Math. Soc., Providence, R.I., 1942.

DePartment of MAThematics, West Virginia UniVersity, Morgantown, West VIRGINIA 26506 